\title{
Vectorcardiography Findings Are Associated with Recurrent Ventricular Arrhythmias and Mortality in Patients with Heart Failure Treated with Implantable Cardioverter-Defibrillator Device
}

\author{
Uzma Chaudhry ${ }^{\mathrm{a}}$ Daniel Cortez ${ }^{\mathrm{b}, \mathrm{c}}$ Pyotr G. Platonov ${ }^{\mathrm{a}}$ Jonas Carlson ${ }^{\mathrm{c}}$ \\ Rasmus Borgquist ${ }^{a}$ \\ a Department of Cardiology, Clinical Sciences, Lund University, Arrhythmia Clinic, Skane University Hospital, \\ Lund, Sweden; ${ }^{b}$ Department of Pediatric Cardiology, University of Minnesota/Masonic Children's Hospital, \\ Minneapolis, MN, USA; ' Department of Cardiology, Clinical Sciences, Lund University, Lund, Sweden
}

\begin{abstract}
Keywords
Spatial QRS-T angle $\cdot$ QRS vector magnitude $\cdot$ Heart failure $\cdot$ T-wave vector magnitude $\cdot$ Implantable cardioverter-defibrillator · Left bundle branch block . Cardiovascular magnetic resonance $\cdot$ Late gadolinium enhancement
\end{abstract}

\begin{abstract}
Background: There is a need for refined risk stratification of sudden cardiac death and prediction of ventricular arrhythmias to correctly identify patients who are expected to benefit the most from implantable cardioverter-defibrillator (ICD) therapy. Methods: We conducted a registry-based retrospective observational study on patients with either ischemic (ICMP) or nonischemic dilated cardiomyopathy (NICMP) treated with ICD between 2002 and 2013 at a tertiary referral center. We evaluated 3 vectorcardiography (VCG) indices; spatial QRS-T angle, QRS vector magnitude (QRSvm), and $T$-wave vector magnitude (Twvm), and their association with all-cause mortality and ventricular arrhythmias. The VCG indices were automatically computed from resting 12lead electrocardiograms before ICD implantation. Results: 178 patients were included in the study; $53.4 \%$ had ICMP, $79.2 \%$ were male, and mean ejection fraction was $27.4 \%$.
\end{abstract}

karger@karger.com www.karger.com/crd

Karger $\stackrel{\text { ' }}{5}$

BOPEN ACCESS
(C) 2020 The Author(s)

Published by S. Karger AG, Basel

This is an Open Access article licensed under the Creative Commons Attribution-NonCommercial-4.0 International License (CC BY-NC) (http://www.karger.com/Services/OpenAccessLicense), applicable to the online version of the article only. Usage and distribution for commercial purposes requires written permission.
During the follow-up (median 89 months), 40 patients (23\%) died; $31 \%$ had appropriate ICD therapy. In multivariate analysis with dichotomized variables, QRS-T angle $>152^{\circ}$ and Twvm $<0.38 \mathrm{mV}$ were significantly associated with increased mortality: HR 2.64 (95\% Cl 1.14-6.12, $p=0.02)$ and HR 5.30 (95\% Cl 2.31-12.11, $p<0.001)$, respectively. QRSvm $<1.54$ $\mathrm{mV}$ was borderline significant with mortality outcome $(p=$ 0.10 ). The composite score of all 3 VCG indices, a score of 3 , conferred an increased risk of mortality (including heart failure mortality) in multivariate analysis: HR $13.80(95 \% \mathrm{Cl} 3.44-$ 55.39, $p<0.001)$. Conclusion: The spatial QRS-T angle and Twvm are emerging VCG indices which are independently associated with mortality in patients with reduced left ventricular ejection fraction due to ICMP or NICMP. Using a composite score of all 3 vector indices, a maximum score was associated with poor long-term survival.

(C) 2020 The Author(s)

Published by S. Karger AG, Basel

\section{Introduction}

Sudden cardiac death is a major disease burden often triggered by ventricular arrhythmias in patients with underlying cardiac disease [1]. Globally, it has been estimated to account for 4-5 million deaths per year [2]. Sur- 
vivors of sudden cardiac arrest are unequivocally treated with an implantable cardioverter-defibrillator device (ICD) $[3,4]$. In addition, patients considered to be at high risk for the development of life-threatening cardiac arrhythmias are provided with an ICD for primary prevention $[3,4]$. Current ICD guidelines are based on randomized clinical trials which primarily focus on reduced left ventricular ejection fraction (LVEF) or a history of ventricular arrhythmia as surrogate markers for sudden cardiac death $[3,4]$. The number needed to treat to save 1 life with ICD therapy is relatively high considering the cost and risk of device complications. A refined risk stratification to predict survival and future ventricular arrhythmias would be useful in order to correctly identify patients who are expected to benefit the most from ICD therapy. Vectorcardiography (VCG) is an important complement to standard 12-lead electrocardiography and is a three-dimensional representation of the cardiac vector loop in 3 orthogonal planes: vertical, transverse, and sagittal [5]. VCG has been reported to be superior to standard ECG in the diagnosis of cardiac disease [5-8]. Novel VCG indices are emerging, which can now be automatically derived. A widened spatial QRS-T angle has been shown to predispose to cardiac mortality and ventricular arrhythmia [9-12]. A reduced QRS vector magnitude (QRSvm) predicts ventricular arrhythmia in selected cohorts [13-15]. A reduced T-wave vector magnitude (Twvm) is a marker for ventricular repolarization changes, and a lower Twvm value has been associated with an increased risk of cardiac events $[16,17]$.

\section{Objectives}

The aim of this project was to evaluate the association between VCG markers, all-cause mortality, and ventricular arrhythmia in a cohort of patients with reduced ejection fraction (EF), who were treated with an ICD.

\section{Methods}

\section{Study Population}

We conducted a registry-based retrospective observational study on a mixed cohort with ischemic dilated cardiomyopathy (ICMP) and non-ICMP (NICMP) at the Skåne University Hospital Lund between 2002 and 2013 who had undergone cardiovascular magnetic resonance (CMR) imaging before ICD implantation, including CRT-D. The indication for ICD was either primary or secondary prevention as per European Society of Cardiology guidelines [4]. Study patients were identified from systematic cross-matching of medical records. Clinical information was obtained from electronic medical records and the National Swedish Pacemaker and Implantable Cardioverter-Defibrillator (ICD) Registry. ICMP was defined as (i) a history of myocardial infarction or revascularization (coronary artery bypass graft or percutaneous coronary intervention), (ii) stenosis $\geq 50 \%$ of the left main artery or $\geq 70 \%$ of the proximal left anterior descending artery, or (iii) $\geq 70 \%$ stenosis of 2 or more epicardial vessels [18]. Patients not fulfilling the criteria for ICMP were classified as NICMP. LVEF was determined by CMR.

Electrocardiography Analysis

Baseline resting 12-lead ECGs acquired before and closest to ICD/CRT-D implantation were analyzed in order to avoid potential effects of cardiac pacing on electrophysiological remodeling. Furthermore, patients with existing pacemaker were excluded from analysis. Each ECG was manually reviewed by electrophysiologists. ECGs with sinus rhythm or rate-controlled atrial arrhythmia were included. ECGs with noise, extrasystoles, incomplete intraventricular block, or right bundle branch block were excluded. ECGs with left bundle branch block (LBBB), however, were included for analysis. LBBB was defined as a QRS width $>120 \mathrm{~ms}$ with broad notched or slurred $R$ wave in leads $I, a V L, V_{5}$, and $V_{6}$ [19]. ECGs were obtained using standard 10-lead placement recorded at $25 \mathrm{~mm} / \mathrm{s}$ speed with $10 \mathrm{~mm} / \mathrm{mV}$ for limb and precordial leads. ECGs were stored digitally on the hospital server and were further processed automatically, blinded to clinical data, using the Glasgow algorithm to define the QRS and T-wave fiducial points (amplitudes, peak, or trough of each waveform) in lead I, II, and $\mathrm{V}_{1}-\mathrm{V}_{6}$ followed by application of Kors' regression-related method to calculate the spatial QRS-T angle and QRSvm and Twvm (Fig. 1) $[20,21]$. The method has been described previously [22].

\section{Study Endpoints}

The primary study endpoint was all-cause mortality. Subanalyses were performed for heart failure mortality and appropriate ICD therapy, or death. ICD data, either from manual interrogation or remotely $\left(\right.$ Merlin ${ }^{\circledR}$, CareLink ${ }^{\circledR}$ or Latitude ${ }^{\circledR}$ ), were obtained from electronic medical records. Appropriate ICD therapy was defined as antitachycardiac pacing or shock due to tachyarrhythmia of ventricular origin. Sustained ventricular arrhythmia was defined as $\geq 30$ s and electrical storm as 3 or more sustained episodes of ventricular arrhythmia or appropriate shocks from an ICD within $24 \mathrm{~h}$. Mortality data were retrieved from the Swedish National Board of Health and Welfare cause of death registry and was cross checked with data in electronic medical records. Endpoints were adjudicated by a senior electrophysiologist.

\section{CMR}

CMR was performed as previously described by Chaudhry et al. [23]. In short, LVEF was determined by cine imaging in breath hold with semiautomatic tracing of the endocardium in both short-axis and long-axis projections. Late gadolinium enhancement (LGE) was used to determine the presence of scars, which were quantified using a semiautomatic algorithm. MRI analysis was performed using the freely available software Segment (Medviso, Lund, Sweden) [24].

\section{Statistical Analysis}

All statistical analyses were performed with IBM SPSS Statistics (version 25; IBM Corporation, Armonk, NY, USA). Categorical variables are presented as frequencies and percentages, and continuous variables are expressed as means (SD) or medians and interquartile ranges (25th and 75 th percentiles), as appropriate. In- 


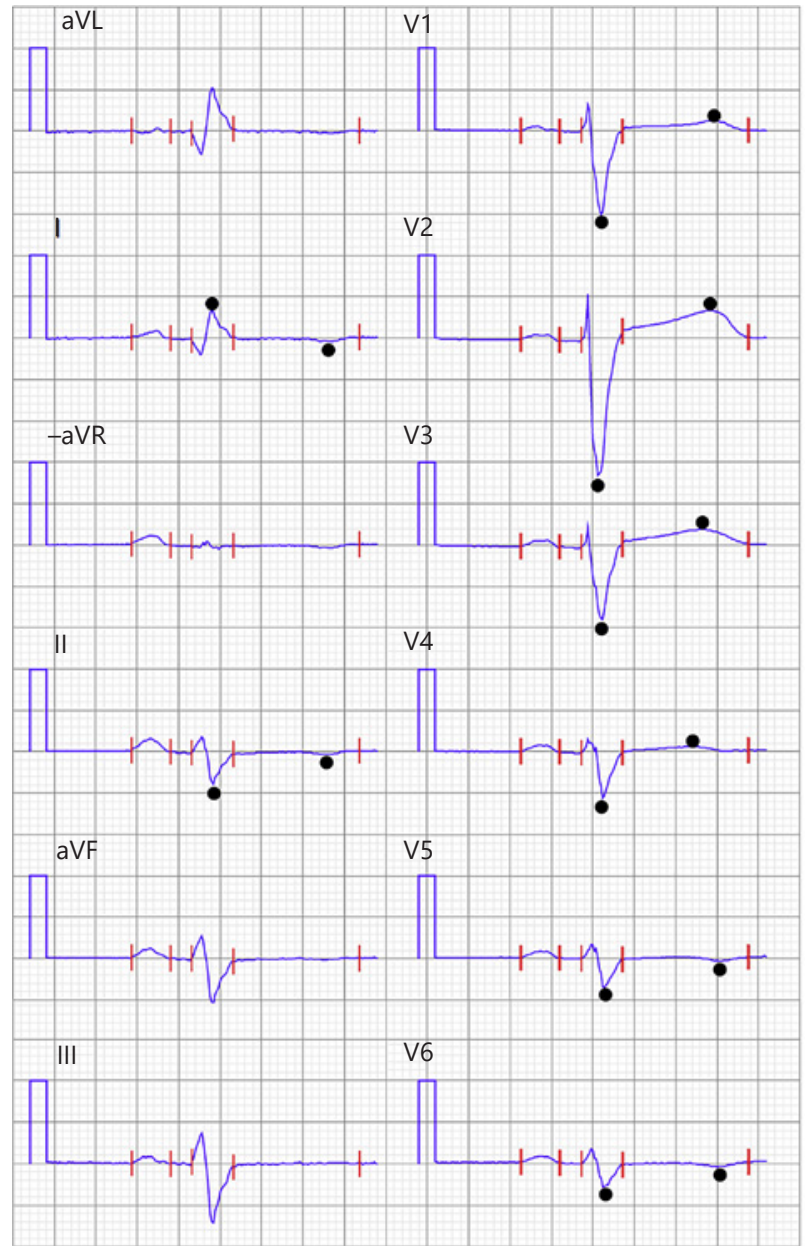

Spatial QRS-T angle $=140^{\circ}$

QRS-vector magnitude $=1.87 \mathrm{mV}$

a T-wave vector magnitude $=0.19 \mathrm{mV}$

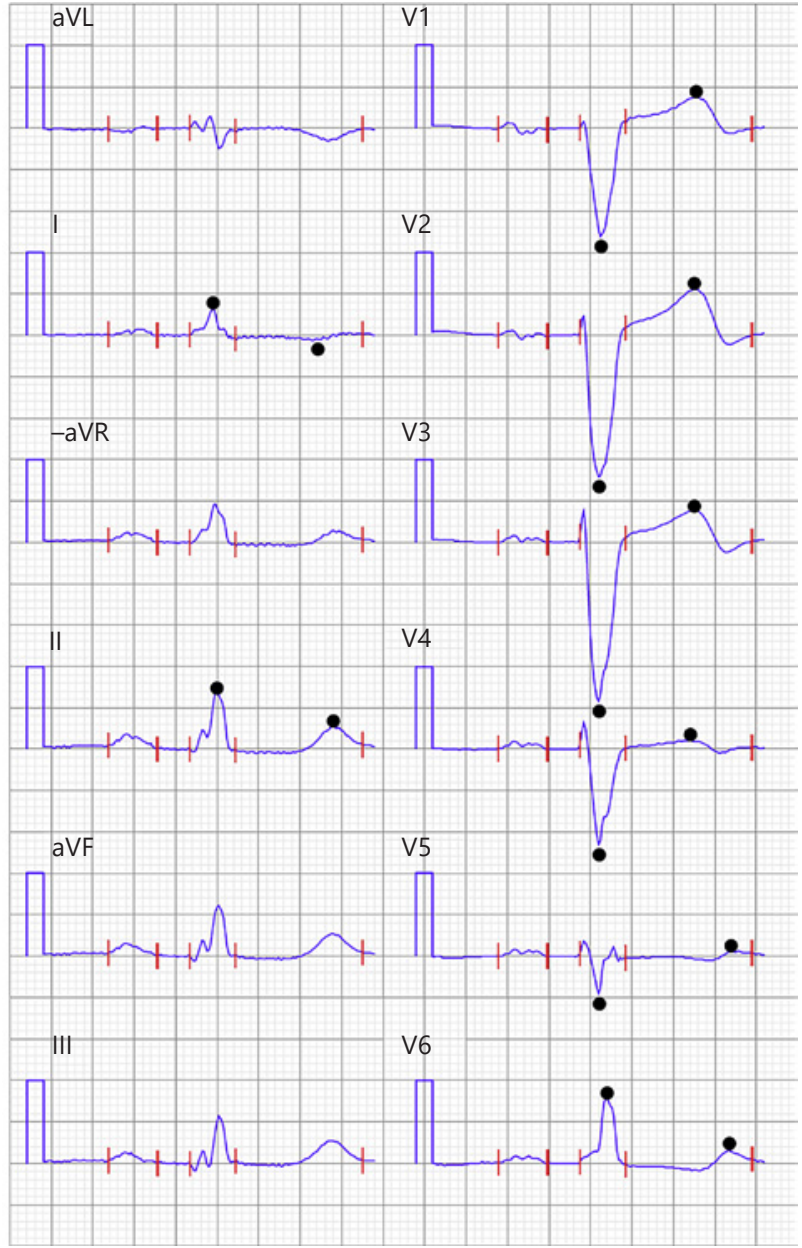

Spatial QRS-T angle $=106^{\circ}$

QRS-vector magnitude $=1.59 \mathrm{mV}$

b $\mathrm{T}$-wave vector magnitude $=0.44 \mathrm{mV}$

Fig. 1. Representative figures of different ECG-derived data. The amplitudes of the illustrated points from the isoelectric line are calculated from 12-lead ECGs and entered into Kors' regression-related method for calculation of QRSvm and Twvm [20]. a Spatial QRS-T angle $=140$, QRSvm $=1.87 \mathrm{mV}$, Twvm $=0.19 \mathrm{mV}$. b Spatial QRS-T angle $=106^{\circ}$, QRSvm $=1.59 \mathrm{mV}$, Twvm $=0.44 \mathrm{mV}$.

ternal correlation between the VCG variables, LBBB pattern, and MRI-verified scar was evaluated by bivariate Spearman correlation test. A correlation $>0.3$ was considered significant. For the primary endpoint, each VCG variable was initially analyzed using timedependent Cox regression analysis in a univariate model. The proportional hazard assumption was tested using a time interaction variable with each of the VCG indices, and the assumption was fulfilled in all cases. In multivariate analysis, prespecified clinically relevant covariates with a previously documented impact on clinical outcome were included $[25,26]$. These variables included age, gender, etiology of heart failure, ICD indication (primary or secondary), EF, QRS duration, LBBB, smoking, and diabetes. KaplanMeier survival curves are presented for all 3 VCG variables (dichotomized, using ROC analysis to select an appropriate cutoff mark), and a combined score variable, with all-cause mortality as outcome. The log rank test was used to test for differences between groups. A $p$ value $<0.05$ was considered statistically significant in all analyses.

\section{Results}

One hundred and seventy-eight patients were included in the study. Complete LGE-CMR scar data were available for analysis in 153 patients. Patient and ECG characteristics are presented in Table 1 . The patients were followed 
Table 1. Patient and ECG characteristics

\begin{tabular}{|c|c|c|c|}
\hline & $\begin{array}{l}\text { All cohort } \\
(n=178)\end{array}$ & $\begin{array}{l}\text { LBBB } \\
(n=103)\end{array}$ & $\begin{array}{l}\text { Narrow QRS } \\
(n=75)\end{array}$ \\
\hline Age, years & $60.5 \pm 13.6$ & $63.7 \pm 11.0$ & $56.2 \pm 15.7$ \\
\hline Males & $141(79.2)$ & $79(76.7)$ & $62(82.7)$ \\
\hline LVEF by CMR & $27.4 \pm 10.9$ & $26.2 \pm 10.4$ & $28.9 \pm 11.4$ \\
\hline NICMP & $83(46.6)$ & $51(49.5)$ & $32(42.7)$ \\
\hline ICMP & $95(53.4)$ & $52(50.5)$ & $43(57.3)$ \\
\hline Secondary prophylactic ICD & $55(30.9)$ & $24(23.3)$ & $31(41.3)$ \\
\hline Diabetes mellitus & $35(19.6)$ & $17(16.5)$ & $18(24)$ \\
\hline Smoker & $40(22.5)$ & $28(27.2)$ & $12(16.0)$ \\
\hline Hypertension & $95(53.4)$ & $58(56.3)$ & $37(49.3)$ \\
\hline \multicolumn{4}{|c|}{ NYHA class before ICD implant } \\
\hline I & $30(16.9)$ & $7(6.8)$ & $23(30.7)$ \\
\hline II & $45(25.3)$ & $25(24.3)$ & $20(26.7)$ \\
\hline III & $91(51.1)$ & $65(63.1)$ & $26(34.7)$ \\
\hline IV & $3(1.7)$ & 0 & $3(4.0)$ \\
\hline CRT-D & $83(46.6)$ & $82(79.6)$ & $1(1.3)$ \\
\hline ICD & $95(53.4)$ & $21(20.4)$ & $74(98.7)$ \\
\hline Follow-up, months & $89(70-119)$ & $82(67-111)$ & $93(79-126)$ \\
\hline Sinus rhythm & $142(79.8)$ & $84(81.6)$ & $58(77.3)$ \\
\hline Heart rate, bpm & $71.0(61-87)$ & $68(58-81)$ & $77 \pm 18$ \\
\hline PR interval, ms & 177 (149-205) & $189 \pm 50$ & $177 \pm 39$ \\
\hline QRS duration, ms & $120(100-156)$ & $156(130-168)$ & $100(92-114)$ \\
\hline QTc duration, ms & $451 \pm 34$ & $463 \pm 33$ & $440 \pm 32$ \\
\hline LBBB & $103(57.9)$ & $103(100)$ & - \\
\hline Spatial QRS-T angle & $151(110-165)$ & $161(148-167)$ & $111(69-145)$ \\
\hline QRSvm & $1.60(1.23-2.07)$ & $1.73(1.39-2.22)$ & $1.47(1.04-1.75)$ \\
\hline Twvm & $0.39(0.25-0.56)$ & $0.49(0.38-0.68)$ & $0.27(0.20-0.38)$ \\
\hline
\end{tabular}

Means (SD), $n$ (\%), or medians (interquartile ranges). See text for abbreviations.

for a median of 89 (IQR 70-119) months after ICD implantation, and no patient was lost to follow-up. Forty patients (23\%) died during the follow-up time. Twenty-eight (16\%) died due to cardiovascular causes, of which $17(61 \%)$ were due to congestive heart failure, and $8(29 \%)$ due to cardiac arrest. Fifty-five patients (31\%) had appropriate ICD therapy for ventricular tachycardia or ventricular fibrillation.

There was a significant bivariate correlation between LBBB and all vector indices; Twvm $(r=0.53)$, QRSvm $(r=0.31)$, and QRS-T angle $(r=0.54)$. All multivariable analyses below, therefore, include adjustment for LBBB, in addition to other clinically relevant parameters.

\section{QRS-T Angle}

A larger QRS-T angle was associated with increased total mortality in univariate analysis: $\mathrm{HR}$ per $10^{\circ} 1.10$ (95\% CI 1.003-1.20, $p=0.04$ ), but it was borderline in multivariate analyses: HR 1.13 (95\% CI 0.997-1.27, $p=$ 0.055). An optimal cutoff value of $152^{\circ}$ was obtained with
ROC curve analysis (AUC 0.58). In multivariate analysis with dichotomized variable, a QRS-T angle $>152^{\circ}$ was associated with increased total mortality, HR 2.64 (95\% CI 1.14-6.12, $p=0.02$; Table 2). In Kaplan-Meier survival analysis with dichotomized variable, QRS-T angle $>152^{\circ}$ was significantly associated with higher total mortality ( $p=0.013)$ and heart failure mortality $(p=0.017)$, but not ICD therapy or death $(p=0.33)$.

\section{QRSvm}

Decreasing QRSvm was borderline associated with increased mortality in univariate analyses: HR 0.96 per 0.1 $\mathrm{mV}$ increase (95\% CI 0.91-1.01, $p=0.09$ ) but not in multivariate analyses $(p=0.18)$. An optimal cutoff value of $1.54 \mathrm{mV}$ was obtained with ROC curve analysis (AUC $0.60)$. In multivariate analysis with dichotomized variable, a QRSvm $<1.54 \mathrm{mV}$ was not associated with increased total mortality ( $p=0.10$; Table 2). In Kaplan-Meier survival analysis with dichotomized variable, QRSvm $<1.54 \mathrm{mV}$ 
Table 2. Univariate and multivariate analyses of the individual VCG variables ${ }^{1}$ and the combined VCG score to predict clinical outcomes

\begin{tabular}{|c|c|c|c|c|c|c|}
\hline & \multicolumn{6}{|c|}{ Total mortality } \\
\hline & \multicolumn{3}{|c|}{ univariate model } & \multicolumn{3}{|c|}{ adjusted model ${ }^{2}$} \\
\hline & $\mathrm{HR}$ & $95 \% \mathrm{CI}$ & $p$ value & $\mathrm{HR}$ & $95 \% \mathrm{CI}$ & $p$ value \\
\hline QRS-T angle $>152^{\circ}$ & 2.20 & $1.17-4.14$ & 0.02 & 2.64 & $1.14-6.12$ & 0.02 \\
\hline QRSvm $<1.54 \mathrm{mV}$ & 1.87 & $0.998-3.51$ & 0.051 & 1.86 & $0.90-3.87$ & 0.10 \\
\hline Twvm $<0.38 \mathrm{mV}$ & 2.50 & $1.30-4.84$ & 0.006 & 5.30 & $2.31-12.11$ & $<0.001$ \\
\hline VCG score 1 vs. 0 & 1.40 & $0.50-3.92$ & 0.53 & 1.18 & $0.40-3.42$ & 0.77 \\
\hline VCG score 2 vs. 0 & 3.37 & $1.24-9.16$ & 0.02 & 2.52 & $0.82-7.72$ & 0.11 \\
\hline VCG score 3 vs. 0 & 6.54 & $1.99-21.47$ & 0.002 & 13.80 & $3.44-55.39$ & $<0.001$ \\
\hline \multicolumn{7}{|c|}{ Heart failure mortality } \\
\hline QRS-T angle $>152^{\circ}$ & 3.35 & $1.17-9.57$ & 0.02 & 2.73 & $0.64-11.5$ & 0.17 \\
\hline QRSvm $<1.54 \mathrm{mV}$ & 1.41 & $0.54-3.66$ & 0.48 & 1.57 & $0.49-5.09$ & 0.45 \\
\hline Twvm $<0.38 \mathrm{mV}$ & 2.19 & $0.81-5.91$ & 0.12 & 13.2 & $2.95-58.89$ & 0.001 \\
\hline VCG score 1 vs. 0 & 2.11 & $0.45-9.96$ & 0.35 & 1.47 & $0.27-8.00$ & 0.66 \\
\hline VCG score 2 vs. 0 & 2.02 & $0.37-11.07$ & 0.42 & 0.88 & $0.12-6.59$ & 0.90 \\
\hline VCG score 3 vs. 0 & 9.24 & $1.54-55.54$ & 0.02 & 15.66 & $1.52-160.88$ & 0.02 \\
\hline \multicolumn{7}{|c|}{ Appropriate ICD therapy or death } \\
\hline QRS-T angle $>152^{\circ}$ & 1.24 & $0.81-1.91$ & 0.33 & 1.24 & $0.72-2.13$ & 0.44 \\
\hline QRSvm $<1.54 \mathrm{mV}$ & 1.30 & $0.85-2.00$ & 0.23 & 1.16 & $0.72-1.85$ & 0.54 \\
\hline Twvm $<0.38 \mathrm{mV}$ & 1.56 & $1.01-2.41$ & 0.04 & 1.63 & $0.97-2.71$ & 0.06 \\
\hline VCG score 1 vs. 0 & 0.79 & $0.44-1.43$ & 0.44 & 0.76 & $0.41-1.41$ & 0.39 \\
\hline VCG score 2 vs. 0 & 1.35 & $0.75-2.46$ & 0.32 & 1.09 & $0.57-2.08$ & 0.80 \\
\hline VCG score 3 vs. 0 & 3.00 & $1.34-6.70$ & 0.007 & 2.50 & $1.02-6.09$ & 0.045 \\
\hline
\end{tabular}

${ }^{1}$ Dichotomized variable obtained using ROC curve analysis. ${ }^{2}$ Adjusted for age, gender, etiology of heart failure, ICD indication (primary or secondary), EF, QRS duration, LBBB, smoking, and diabetes.

was significantly associated with higher mortality $(p=$ 0.047 ; Fig. 2 b), but not heart failure mortality $(p=0.48)$ and appropriate ICD therapy or death $(p=0.23)$.

\section{Twvm}

A decreasing Twvm was borderline associated with increased mortality in univariate analysis: HR 0.99 per $0.01-\mathrm{mV}$ increase $(95 \%$ CI $0.9-1.00, p=0.06)$ and in multivariate analysis with a significant HR of 0.98 (95\% CI 0.96-0.997, $p=0.03)$. An optimal cutoff mark of 0.38 $\mathrm{mV}$ was obtained using ROC curve analysis (AUC 0.63). In multivariate analysis with dichotomized variable, Twvm $<0.38 \mathrm{mV}$ was significantly associated with higher mortality: HR 5.30 (95\% CI 2.31-12.11, $p<0.001$; Table 2). In Kaplan-Meier survival analysis with dichotomized variable, Twvm $<0.38 \mathrm{mV}$ was associated with increased total mortality $(p=0.005)$ and appropriate ICD therapy or death $(p=0.04)$, but not with heart failure mortality $(p=0.11)$.

\section{Combined Score of All 3 VCG Indices}

A combined score was constructed using all 3 VCG variables dichotomized using the ROC curve-derived cutoff marks. One point was allocated to each VCG variable (QRS-T angle $>152^{\circ}$, QRSvm $<1.54 \mathrm{mV}$, and Twvm $<0.38 \mathrm{mV}$ ), the possible scores ranging from 0 to 3 points. A higher score was significantly correlated with higher mortality both in univariate $(p=0.001)$, and multivariate analysis $(p<0.001$; Table 2$)$. Similarly, in Kaplan-Meier survival analysis, a higher score was associated with increased all-cause mortality, heart failure death, and appropriate ICD therapy or death (Fig. 3). Patients with a maximum score of 3 points had a particularly poor longterm survival.

\section{VCG Indices and Scar}

In patients with LGE-CMR-verified scar $(n=109)$, there was a significant negative correlation between more scar and Twvm: correlation coefficient $r=-0.36$ ( $p<$ $0.001)$ and QRSvm $r=-0.40(p<0.001)$, respectively. A 


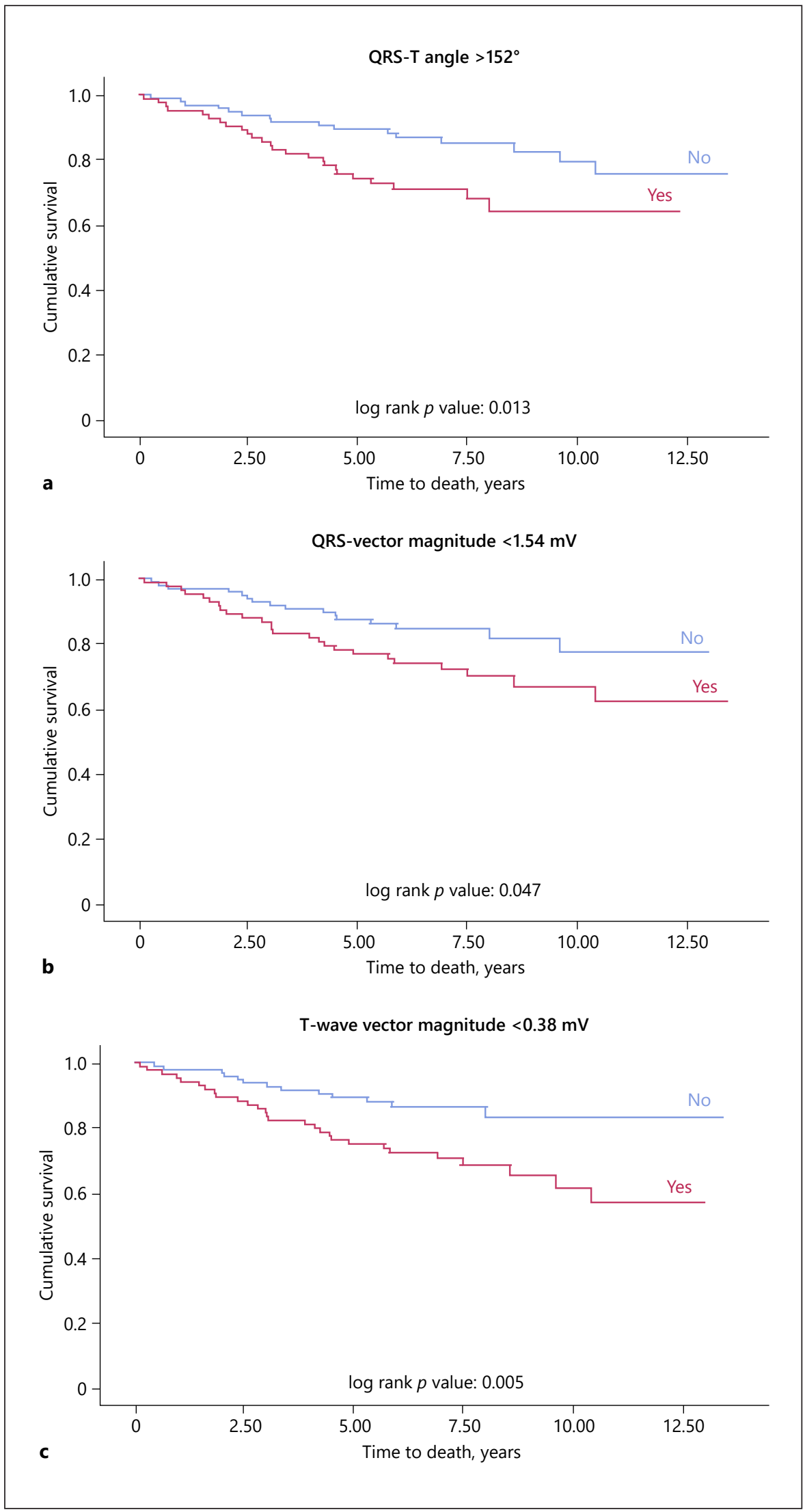

Fig. 2. Kaplan-Meier curves for the individual VCG variables and all-cause mortality. a QRS-T angle $>152^{\circ}$. b QRSvm $<1.54$ $\mathrm{mV}$. c Twvm $<0.38 \mathrm{mV}$. 

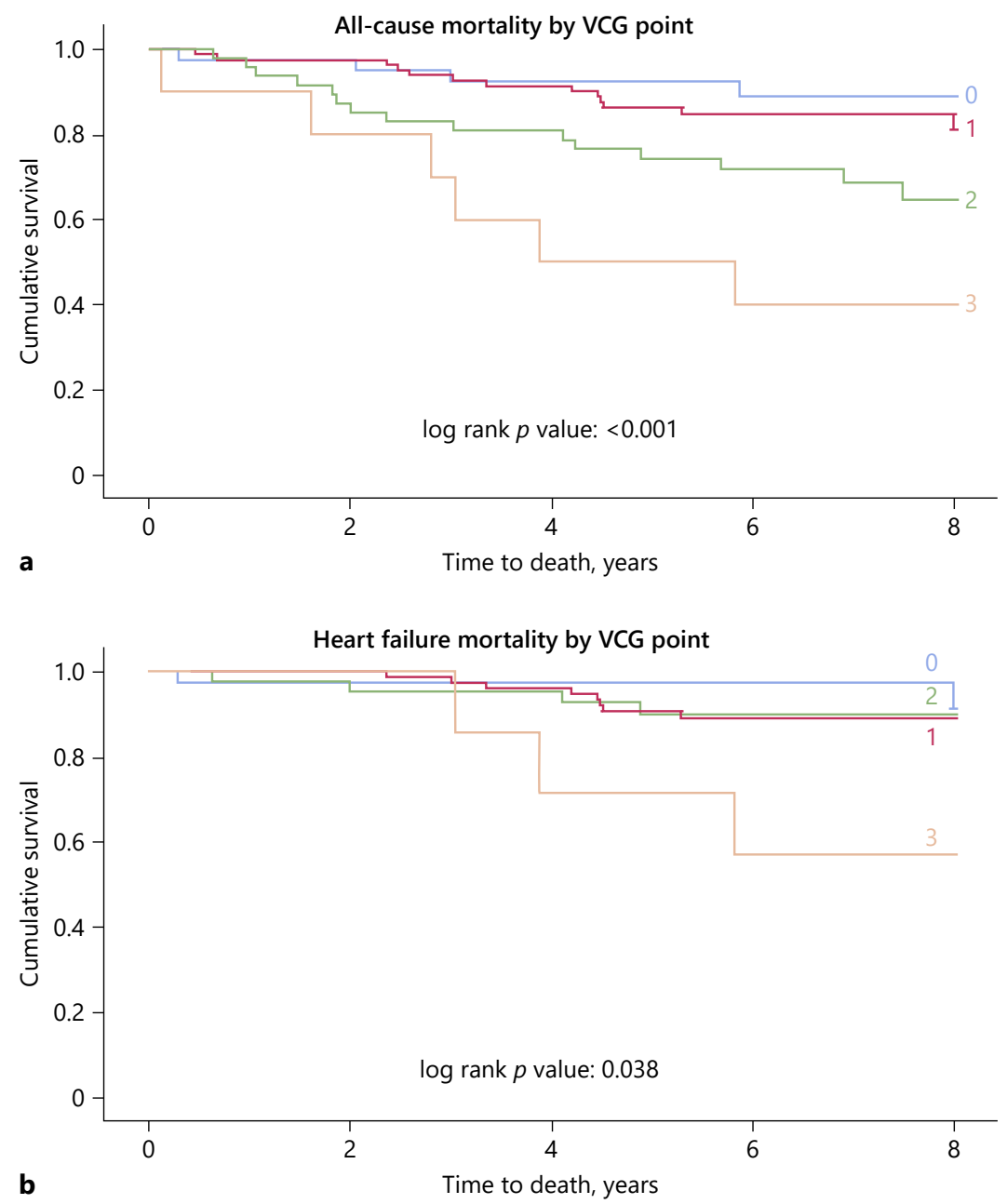

Fig. 3. Kaplan-Meier curve of the VCG score and its association to all-cause mortality (a), heart failure mortality (b), and appropriate ICD therapy or death (c).

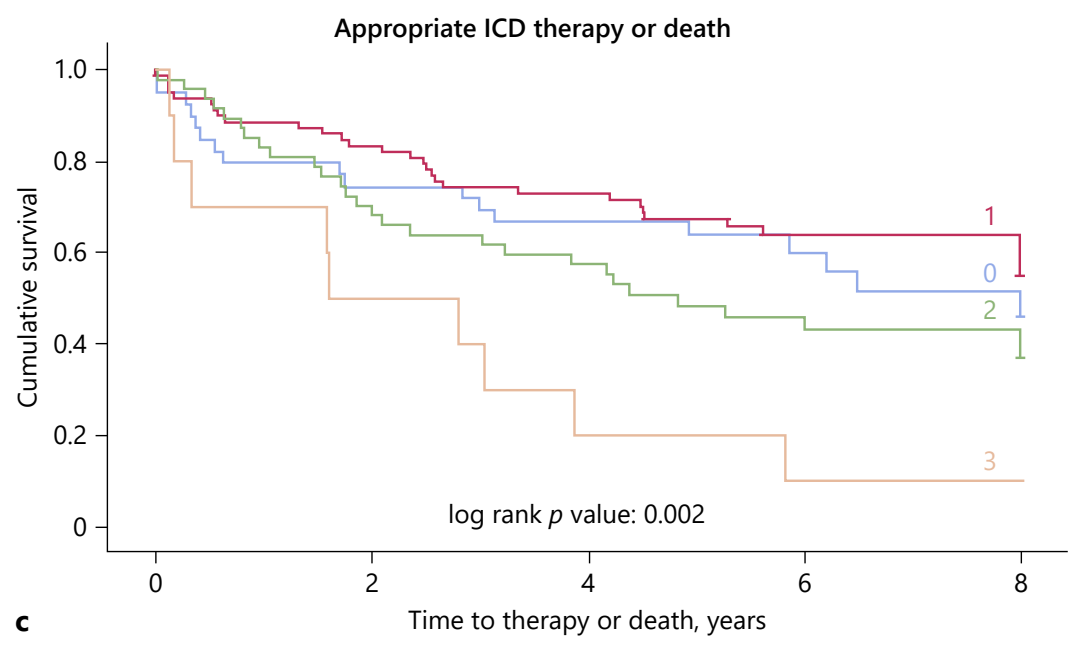


similar statistically significant relation was not observed with QRS-T angle, neither in the entire material nor in the subanalysis for ICMP only. Increasing scar volume did not independently predict higher mortality, appropriate ICD therapy, or the combined endpoint.

\section{Discussion}

Our study showed that wider spatial QRS-T angle and reduced Twvm were associated with higher mortality in a mixed patient cohort with ICMP and NICMP. Furthermore, using a composite score of all 3 vector indices (also including QRSvm), we were able to show a strong association with increased all-cause mortality, as well as heart failure mortality, and appropriate ICD therapy or death. Even though scar burden (measured by LGE-CMR) was associated with reduced QRSvm and Twvm, the VCG indices conferred additional information associated with clinical outcome, death, or appropriate ICD therapy. Our study findings are in keeping with previous studies and expand on the prognostic utility of ECG indices $[9,11,13$, 15, 27-30]. The association between VCG indices and allcause and heart failure mortality may have prognostic utility for patients eligible for ICD therapy.

\section{QRS-T Angle}

The spatial QRS-T angle is the angle between the mean spatial QRS vector and the mean spatial T vector, that is, the angle between ventricular depolarization and repolarization vectors [10]. In a normal myocardium, the QRS and $\mathrm{T}$-vector loops point in the same spatial direction, but with changes in ventricular depolarization and repolarization, the vectors point in opposite spatial directions $[10,27]$. In our study, a spatial QRS-T angle $>152^{\circ}$ correlated with mortality $(p=0.005)$, especially heart failure deaths. There are, however, no standardized reference values to separate normal QRS-T angles from abnormal ones, and our ROC-based analysis showed a relatively weak correlation to mortality (AUC 0.58). Largely, existing evidence is from population-based studies, which all have suggested different values, with normal values in the range of $0-50^{\circ}$ and $0-105^{\circ}$ and abnormal values described as both $100-180^{\circ}$ and $135-180^{\circ}[9,11,27]$, and in relation to which our cutoff mark also falls within the abnormal spectrum. In large population-based studies, a widened QRS-T angle has been shown to confer an increased risk of cardiovascular death $[9,11]$. In our study, a QRS-T angle $>152^{\circ}$ did confer a near 3-fold increased risk of mortality. In comparison, a QRS-T angle $>135^{\circ}$ has been

Vectorcardiography to Predict Outcome of Patients with Ventricular Arrhythmia shown to predict a 5 -fold increased risk of cardiac death (independent of conventional cardiovascular risk factors) [9]. Our results did not show an association between widened QRS-T angle and appropriate ICD therapy, in contrast to the study by Borleffs et al. [12] who found a 7-fold increased risk of appropriate ICD therapy in an ICMP cohort with QRS-T angle $>100^{\circ}$.

\section{QRSvm}

The QRSvm is a measurement of depolarization voltage dispersion (estimated QRS amplitude peaks or troughs). Studies are sparse, but it is hypothesized that the dispersion of depolarization results in reduced unidirectional forces, thereby yielding a lower QRS peak magnitude [13]. A QRSvm cutoff level of $1.54 \mathrm{mV}$ was neither predictive of nor associated with mortality. Previously, cutoffs of 1.31 and $1.55 \mathrm{mV}$ have been described for selected cohorts with tetralogy of Fallot and Brugada syndrome, respectively $[13,15]$. A lower QRSvm value did not confer an increased risk of appropriate ICD therapy in our study, which has been reported in patients with Brugada syndrome previously [15]. Up to now, published data on patients with congestive heart failure are still lacking; only in patients with tetralogy of Fallot, a lower QRSvm was associated with the development of ventricular tachyarrhythmias [14] and perioperative atrial tachycardias [13]. In view of our borderline QRSvm findings, one can only speculate if the marker is perhaps better suited for the assessment of right- than left-heart pathology, which was predominant in our study.

\section{Twvm}

Twvm is defined as the maximum distance from the origin of the three-dimensional T-wave loop. It is computed from the amplitudes of the T-wave from 3 orthogonal planes and thus avoids potential bias or underestimation resulting from rotation of the main vector. We show that a decreasing Twvm is independently associated with death $(p=0.015)$. A Twvm $<0.38$ and $<0.24 \mathrm{mV}$ was in our study significantly associated with higher mortality in all cohort and narrow QRS subgroups, respectively. A previous study has shown that Twvm in narrow QRS correlates to hypertensive ventricular repolarization changes compared to a control group without hypertension, but the study did not evaluate any clinical endpoints [16]. However, a cutoff level equivalent to the one in our study, for patients with narrow QRS, was identified: $0.24 \mathrm{mV}$ [16]. A recent study showed that Twvm $<0.30 \mathrm{mV}$ conferred a threefold increased risk of cardiac events in females with long QT syndrome (genotype-positive $\mathrm{KCNH} 2$ with nor- 
mal QTc values) [17]. More studies, however, have evaluated frontal T-wave amplitudes, as opposed to the possibly superior three-dimensional vector used in our study. In heterogenous ICD cohorts, frontal T-wave amplitudes did predict appropriate ICD therapy [29, 30].

\section{VCG Score Utility}

We have shown that the composite score of all 3 VCG indices confers an increased risk of mortality, including death from heart failure, and appropriate ICD therapy or death. In both univariate and multivariate analyses, a single VCG point compared to zero points was not predictive of mortality. However, in univariate analysis, a VCG score $\geq 2$ compared to zero points was associated with a 3 - to 7-fold increased risk of all-cause mortality, and in multivariate analysis a VCG score of 3 compared to zero points was associated with a near 14-fold increased risk of mortality. Our results suggest that the VCG indices assessed are representative of malignant electrical disturbances predictive of more advanced disease and an increased risk of death. Thus, the combined VCG score (which considers different markers for depolarization and repolarization) could be useful for providing additive prognostic information in patients with reduced EF.

\section{VCG Indices and Scar}

A myocardial scar is likely to influence ventricular depolarization due to shift and dispersion of QRS vectors during depolarization of viable myocardium around the scar [31]. One can hypothesize that increased scar burden is associated with myocyte loss (reduced myocardium to depolarize) and hence smaller vectors. In our study, a negative, but significant correlation was observed with lower QRSvm and Twvm and scar burden. Despite QRS$T$ angle being a marker for both structural and ionic channel changes, in our study there was no correlation between widened QRS-T angle and scar burden (including a subanalysis for ICMP patients). This is in contrast to a study by Shi et al. [32], where a scar of ischemic etiology was associated with a wider QRS-T angle.

\section{Limitations}

We have conducted a single-center retrospective observational study, and, therefore, the generalizability of our findings may be limited. Our cohort is mixed and included patients with ICMP and NICMP. No patient was lost to follow-up, but the sample size was relatively small, which may have affected the power of our study. Our study population had an overrepresentation of patients with CRT-D, as our hospital was conducting a prospective study on CRT patients, which also had CMR investigation in the study protocol. This also explains the high number of patients with LBBB in our study. The best reference values for the VCG indices are not known and warrant further validation. Our proposed cutoff values were based on ROC analysis, but ideally the VCG indices should be further assessed in a larger ICD study population comparing subjects with narrow QRS and LBBB in order to determine the impact of $\mathrm{LBBB}$ on QRS and Twave vector dispersion. During $\mathrm{LBBB}$, the left ventricle is activated at a slower rate and with a different QRS axis vector compared to normal Purkinje conduction system activation. It is, therefore, possible that LBBB causes significant depolarization and repolarization changes that may affect the prognostic associations of the VCG variables compared to narrow QRS complexes.

The ECGs analyzed were recorded before device implantation, that is, VCG values obtained during intrinsic QRS activation and before ICD implantation. The ECGs used in the study were representative of the disease stage at the time, but one needs to bear in mind that both ECG changes and cardiac disease processes are dynamic.

\section{Conclusion}

We show that VCG-derived spatial QRS-T angle and Twvm are associated with mortality in patients with reduced LVEF due to ICMP or NICMP. These variables can be automatically computed from standard 12-lead ECG. Using a composite VCG-derived score, these ECG-based measures provide incremental prognostic value in patients with known cardiac disease, and thus may have a role in risk prediction models for patients eligible for ICD treatment. The score requires further validation in larger prospective studies.

\section{Statement of Ethics}

The study was approved by the local ethics committee and complies with the Declaration of Helsinki. Requirement of informed consent was waived by the ethics committee due to the retrospective nature of the study and the rather large patient cohort.

\section{Conflict of Interest Statement}

The authors have no conflicts of interest to declare. 


\section{Funding Sources}

Governmental funding of clinical research within the Swedish National Health Service (ALF) and Region Skåne research grant covered salary costs.

\section{Author Contributions}

U.C.: overall responsible for the project including data collection and analysis and manuscript writing. D.C.: responsible for VCG analyses. P.G.P.: conception of the idea and supervision of the project. J.C.: responsible for ECG database and digital transformation of ECGs enabling VCG calculations. R.B.: conception of the idea, supervision of the project, data analysis, and manuscript writing.

\section{References}

1 Al-Khatib SM, Stevenson WG, Ackerman MJ, Bryant WJ, Callans DJ, Curtis AB, et al. 2017 AHA/ACC/HRS guideline for management of patients with ventricular arrhythmias and the prevention of sudden cardiac death: Executive summary: a report of the American College of Cardiology/American Heart Association Task Force on Clinical Practice Guidelines and the Heart Rhythm Society. Heart Rhythm. 2018 Oct;15(10): e190-252.

2 Chugh SS, Reinier K, Teodorescu C, Evanado A, Kehr E, Al Samara M, et al. Epidemiology of sudden cardiac death: clinical and research implications. Prog Cardiovasc Dis. 2008 NovDec;51(3):213-28.

3 Epstein AE, DiMarco JP, Ellenbogen KA, Estes NA 3rd, Freedman RA, Gettes LS, et al; American College of Cardiology/American Heart Association Task Force on Practice Guidelines (Writing Committee to Revise the ACC/AHA/NASPE 2002 Guideline Update for Implantation of Cardiac Pacemakers and Antiarrhythmia Devices); American Association for Thoracic Surgery; Society of Thoracic Surgeons. ACC/AHA/HRS 2008 guidelines for device-based therapy of cardiac rhythm abnormalities: a report of the American College of Cardiology/American Heart Association Task Force on Practice Guidelines (Writing Committee to Revise the ACC/ AHA/NASPE 2002 Guideline Update for Implantation of Cardiac Pacemakers and Antiarrhythmia Devices) developed in collaboration with the American Association for Thoracic Surgery and Society of Thoracic Surgeons. J Am Coll Cardiol. 2008 May; 51(21):e1-62.

4 Brignole M, Auricchio A, Baron-Esquivias G, Bordachar P, Boriani G, Breithardt OA, et al; European Society of Cardiology (ESC); European Heart Rhythm Association (EHRA). 2013 ESC guidelines on cardiac pacing and cardiac resynchronization therapy: the Task Force on Cardiac Pacing and Resynchronization Therapy of the European Society of Cardiology (ESC). Developed in collaboration with the European Heart Rhythm Association (EHRA). Europace. 2013 Aug;15(8):1070118.

5 Jaros R, Martinek R, Danys L. Comparison of different electrocardiography with vectorcardiography transformations. Sensors (Basel). 2019 Jul;19(14):E3072.

Vectorcardiography to Predict Outcome of Patients with Ventricular Arrhythmia
6 Pérez Riera AR, Uchida AH, Filho CF, Meneghini A, Ferreira C, Schapacknik E, et al. Significance of vectorcardiogram in the cardiological diagnosis of the 21st century. Clin Cardiol. 2007 Jul;30(7):319-23.

7 Schreck DM, Fishberg RD. Derivation of the 12-lead electrocardiogram and 3-lead vectorcardiogram. Am J Emerg Med. 2013 Aug; 31(8):1183-90.

8 Yang H, Bukkapatnam ST, Komanduri R. Spatiotemporal representation of cardiac vectorcardiogram (VCG) signals. Biomed Eng Online. 2012 Mar;11(1):16.

9 Kardys I, Kors JA, van der Meer IM, Hofman A, van der Kuip DA, Witteman JC. Spatial QRS-T angle predicts cardiac death in a general population. Eur Heart J. 2003 Jul;24(14): 1357-64.

10 Oehler A, Feldman T, Henrikson CA, Tereshchenko LG. QRS-T angle: a review. Ann Noninvasive Electrocardiol. 2014 Nov;19(6):53442.

11 Yamazaki T, Froelicher VF, Myers J, Chun S, Wang P. Spatial QRS-T angle predicts cardiac death in a clinical population. Heart Rhythm. 2005 Jan;2(1):73-8.

12 Borleffs CJ, Scherptong RW, Man SC, van Welsenes GH, Bax JJ, van Erven L, et al. Predicting ventricular arrhythmias in patients with ischemic heart disease: clinical application of the ECG-derived QRS-T angle. Circ Arrhythm Electrophysiol. 2009 Oct;2(5):54854.

13 Cortez D, Barham W, Ruckdeschel E, Sharma $\mathrm{N}$, McCanta AC, von Alvensleben J, et al. Noninvasive predictors of ventricular arrhythmias in patients with tetralogy of Fallot undergoing pulmonary valve replacement. JACC Clin Electrophysiol. 2017 Feb;3(2): $162-70$.

14 Cortez D, Ruckdeschel E, McCanta AC, Collins K, Sauer W, Kay J, et al. Vectorcardiographic predictors of ventricular arrhythmia inducibility in patients with tetralogy of Fallot. J Electrocardiol. 2015 Mar-Apr;48(2): 141-4.

15 Ragab AA, Houck CA, van der Does LJ, Lanters EA, Muskens AJ, de Groot NM. QRS vector magnitude as predictor of ventricular arrhythmia in patients with Brugada syndrome. Am J Cardiol. 2019 Jun;123(12):1962-6.

16 Dilaveris P, Gialafos E, Poloniecki J, Hnatkova K, Richter D, Andrikopoulos G, et al. Changes of the T-wave amplitude and angle: an early marker of altered ventricular repolarization in hypertension. Clin Cardiol. 2000 Aug;23(8):600-6.

17 Cortez D, Zareba W, McNitt S, Polonsky B, Rosero SZ, Platonov PG. Quantitative T-wave morphology assessment from surface ECG is linked with cardiac events risk in genotypepositive $\mathrm{KCNH} 2$ mutation carriers with normal QTc values. J Cardiovasc Electrophysiol. 2019 Dec;30(12):2907-13.

18 Felker GM, Shaw LK, O'Connor CM. A standardized definition of ischemic cardiomyopathy for use in clinical research. J Am Coll Cardiol. 2002 Jan;39(2):210-8.

19 Surawicz B, Childers R, Deal BJ, Gettes LS, Bailey JJ, Gorgels A, et al; American Heart Association Electrocardiography and Arrhythmias Committee, Council on Clinical Cardiology; American College of Cardiology Foundation; Heart Rhythm Society; Endorsed by the International Society for Computerized Electrocardiology. AHA/ACCF/HRS recommendations for the standardization and interpretation of the electrocardiogram: part III: intraventricular conduction disturbances: a scientific statement from the American Heart Association Electrocardiography and Arrhythmias Committee, Council on Clinical Cardiology; the American College of Cardiology Foundation; and the Heart Rhythm Society. J Am Coll Cardiol. 2009 Mar;53(11):976-81.

20 Kors JA, van Herpen G, Sittig AC, van Bemmel JH. Reconstruction of the Frank vectorcardiogram from standard electrocardiographic leads: diagnostic comparison of different methods. Eur Heart J. 1990 Dec;11(12): 1083-92.

21 Macfarlane PW. The University of Glasgow (Uni-G) ECG analysis program. Comput Cardiol. 2005;32:451-4.

22 Tran TV, Cortez D. Clinical application of the QRS-T angle for the prediction of ventricular arrhythmias in patients with the Fontan palliation. Pediatr Cardiol. 2017 Jun;38(5):104956.

23 Chaudhry U, Platonov PG, Jablonowski R, Couderc JP, Engblom H, Xia X, et al. Evaluation of the ECG based Selvester scoring method to estimate myocardial scar burden and predict clinical outcome in patients with left bundle branch block, with comparison to late gadolinium enhancement CMR imaging. Ann Noninvasive Electrocardiol. 2017 Sep; 22(5):e12440 
24 Heiberg E, Sjögren J, Ugander M, Carlsson M, Engblom H, Arheden H. Design and validation of Segment-freely available software for cardiovascular image analysis. BMC Med Imaging. 2010 Jan;10(1):1.

25 Moss AJ, Zareba W, Hall WJ, Klein H, Wilber DJ, Cannom DS, et al; Multicenter Automatic Defibrillator Implantation Trial II Investigators. Prophylactic implantation of a defibrillator in patients with myocardial infarction and reduced ejection fraction. N Engl J Med. 2002 Mar;346(12):877-83.

26 Goldenberg I, Jonas M, Tenenbaum A, Boyko V, Matetzky S, Shotan A, et al; Bezafibrate Infarction Prevention Study Group. Current smoking, smoking cessation, and the risk of sudden cardiac death in patients with coronary artery disease. Arch Intern Med. 2003 Oct;163(19):2301-5.
27 Strauss DG, Mewton N, Verrier RL, Nearing BD, Marchlinski FE, Killian T, et al. Screening entire health system ECG databases to identify patients at increased risk of death. Circ Arrhythm Electrophysiol. 2013 Dec;6(6): 1156-62.

28 Kors JA, Kardys I, van der Meer IM, van Herpen G, Hofman A, van der Kuip DA, et al. Spatial QRS-T angle as a risk indicator of cardiac death in an elderly population. J Electrocardiol. 2003;36 Suppl:113-4.

29 Sugrue A, Killu AM, DeSimone CV, Chahal AA, Vogt JC, Kremen V, et al. Utility of Twave amplitude as a non-invasive risk marker of sudden cardiac death in hypertrophic cardiomyopathy. Open Heart. 2017 Feb;4(1): e000561.
30 Shi B, Harding SA, Jimenez A, Larsen PD. Standard 12-lead electrocardiography measures predictive of increased appropriate therapy in implantable cardioverter defibrillator recipients. Europace. 2013 Jun;15(6): 892-8.

31 Al-Khatib SM, Fonarow GC, Joglar JA, Inoue LY, Mark DB, Lee KL, et al. Primary prevention implantable cardioverter defibrillators in patients with nonischemic cardiomyopathy: a meta-analysis. JAMA Cardiol. 2017 Jun;2(6): 685-8.

32 Shi B, Ferrier KA, Sasse A, Harding SA, Larsen PD. Correlation between vectorcardiographic measures and cardiac magnetic resonance imaging of the left ventricle in an implantable cardioverter defibrillator population. J Electrocardiol. 2014 Jan-Feb;47(1):52-8. 